\title{
A novel fluorescence technique for identification of the pulmonary segments by using the photodynamic diagnosis endoscope system: An experimental study in ex vivo porcine lung
}

\author{
Ryuichi Waseda, MD, Makoto Oda, MD, Isao Matsumoto, MD, Masaya Takizawa, MD, \\ Mitsutaka Suzuki, MD, Masahiro Ohsima, MD, and Go Watanabe, MD
}

\begin{abstract}
Objective: Photodynamic diagnosis is a technique that has been proposed to enhance tumor detection and resection. We modified this technique to identify pulmonary segments and examine the feasibility of this technique in ex vivo porcine lungs.

Methods: The photodynamic diagnosis endoscope system consisted of the D-Light system as the excitation light source and a TRICAM camera as the fluorescence sensing endoscope (Karl Storz GmbH \& Co, Tuttlingen, Germany). Vitamin $B_{2}$ was used as the fluorescence substance. Two kinds of segmentectomy were performed in right porcine lung. After identification of the segmental bronchus, the fluorescent substance was injected transbronchially. The fluorescent segment was observed using the photodynamic diagnosis endoscope system, and the identified intersegmental plane was cut using scissors. The operative data collected were the success rate of accurately identifying the pulmonary segments. The duration and light intensity of fluorescence of the target segment were recorded to provide an objective measurement of success. The same parameters were also measured for the adjacent segment.
\end{abstract}

Results: Overall, 20 segmentectomies were performed, 10 of each kind-cranial segmentectomy and L2 segmentectomy. In all procedures, it was possible to identify the target segment by its yellow-green fluorescence. No unexpected injuries of the major segmental bronchi and vessels nor incorrect recognition of target segments occurred. The success rate of accurately identifying pulmonary segments was $100 \%$. The duration and light intensity of this fluorescence technique was enough to perform pulmonary segmentectomy.

Conclusions: This florescence technique using vitamin $\mathrm{B}_{2}$ and the photodynamic diagnosis endoscope system is useful to identify the target pulmonary segment easily and clearly in ex vivo porcine lung. (J Thorac Cardiovasc Surg 2013;146:222-7)

With an increasing number of patients having smallsized non-small cell lung cancer tumors, anatomic pulmonary segmentectomy has proved to be a valuable operative method for general thoracic surgeons. ${ }^{1,2}$ The identification of the intersegmental plane is essential for a successful anatomic pulmonary segmentectomy, but this identification is often difficult without an imaging method. Several previous reports have documented various methods for identifying the intersegmental plane during pulmonary segmentectomy. ${ }^{3-6}$ Some surgeons have adopted the air inflation-deflation technique. ${ }^{3,4}$ Although this technique makes it easy to identify the intersegmental plane on the lung surface, it may be

From the Department of General and Cardiothoracic Surgery, Kanazawa University, Kanazawa, Ishikawa, Japan.

Disclosures: Authors have nothing to disclose with regard to commercial support.

Received for publication June 28, 2012; revisions received Aug 17, 2012; accepted

for publication Sept 12, 2012; available ahead of print Oct 18, 2012.

Address for reprints: Ryuichi Waseda, MD, General and Cardiothoracic Surgery,

Kanazawa University School of Medicine, Kanazawa, Ishikawa 920-8641, Japan

(E-mail: wryuichi@med.kanazawa-u.ac.jp).

$0022-5223 / \$ 36.00$

Copyright (c) 2013 by The American Association for Thoracic Surgery

http://dx.doi.org/10.1016/j.jtcvs.2012.09.010 difficult to identify the intersegmental plane inside the lung parenchyma when cutting the segment. Furthermore, during video-assisted thoracoscopic surgery (VATS) or minimal access open surgery, an inflated lung may obstruct the exposure and limit the visualization of the target segment.

Photodynamic diagnosis (PDD) is a technique that has been proposed to enhance tumor detection and resection. ${ }^{7}$ The principle of PDD is based on the interaction between a photosensitizing agent with a high uptake by tumor cells and light with an appropriate wavelength, which is absorbed by the agent and reemitted with a different wavelength. In the present study, we modified this PDD technique to identify pulmonary segments instead of tumors.

The ideal method for the identification of the intersegmental plane should allow for accurate and clear identification, not only on the lung surface but also inside the lung parenchyma. It should be easy and safe and be applicable to various surgical fields (ie, VATS and minimal access open surgery). To develop an ideal method, we examined a novel fluorescence technique for the identification of the pulmonary segment using the PDD endoscope system in ex vivo porcine lungs. 


$$
\begin{aligned}
& \text { Abbreviations and Acronyms } \\
& \begin{aligned}
\text { D } & =\text { dorsal } \\
\text { FAD } & =\text { flavin adenine dinucleotide } \\
\text { ICG } & =\text { indocyanine green } \\
\text { L } & =\text { lateral } \\
\text { PDD } & =\text { photodynamic diagnosis } \\
\text { V } & =\text { ventral } \\
\text { VATS } & =\text { video-assisted thoracoscopic surgery }
\end{aligned}
\end{aligned}
$$

\section{METHODS}

\section{PDD Endoscope System}

In the PDD technique, the key instruments are a powerful light source for fluorescence excitation and a special fluorescence sensing endoscope. We used the D-Light system as the excitation light source and a TRICAM camera as the fluorescence sensing endoscope (KARL STORZ GmbH \& Co, Tuttlingen, Germany) (Figure 1). The D-Light system emits light of wavelength 375 to $450 \mathrm{~nm}$ (near ultraviolet). In the TRICAM camera suitable for fluorescence imaging, the wavelength-dependent sensitivity is modified, thereby achieving greater sensitivity of fluorescence than that of a standard camera. In addition, this PDD endoscope system can perform not only fluorescence imaging but also normal light imaging.

\section{Fluorescence Substance}

Vitamin $B_{2}$ was used as the fluorescence substance. $\mathrm{We}^{8}$ previously reported the feasibility and safety of vitamin $\mathrm{B}_{2}$ as a fluorescence substance for pulmonary sentinel node navigation. Vitamin $B_{2}$ is water soluble, nonallergic, and nontoxic. ${ }^{9}$ It emits a strong yellow-green fluorescence when exposed to light with a wavelength of approximately $400 \mathrm{~nm}$. In humans, it is transformed to flavin mononucleotide and flavin adenine dinucleotide (FAD) via the action of cellular enzymes, with the majority becoming FAD sodium. Therefore, FAD sodium (Fladd injection; Taiho Pharmaceutical Co, Ltd, Tokyo, Japan) was used.

\section{Pulmonary Segmentectomy in Ex Vivo Porcine Lungs}

Ex vivo lungs obtained from slaughtered $50-\mathrm{kg}$ pigs were used. The right porcine lung consists of the cranial, middle, caudal, and accessory lobes. ${ }^{10}$ Two kinds of segmentectomy were performed. The first was a cranial segmentectomy in the right cranial lobe, assumed to be a typical and easy segmentectomy, and the second was the lateral (L2) segmentectomy in the right caudal lobe, assumed to be an atypical and a difficult segmentectomy (Figure 2, $A$ and $B$ ). The cranial segment has only 1 intersegmental plane adjacent to the caudal segment in the right cranial lobe; the L2 segment has 3 intersegmental planes adjacent to the lateral, dorsal, and ventral (L3, D2, and V2) segments in the right caudal lobe.

The surgical procedure was performed as follows. Initially, each segmental bronchus was exposed and identified. After the identified segmental bronchus had been cut off, a plastic catheter was inserted into the cut bronchus, followed by ligation of the root of the bronchus. Vitamin $\mathrm{B}_{2}$ solution diluted with saline was injected slowly and steadily by using a manual syringe. The concentration of FAD sodium was $0.4 \mathrm{mg} / \mathrm{mL}$. Subsequently, the fluorescent segment was observed using the PDD endoscope system. After sufficient solution had been injected such that the fluorescence was observed in peripheral lung parenchyma, the catheter was removed and the bronchus was ligated. Lung parenchyma was cut using scissors along the identified intersegmental plane.

All experimental procedures were performed in accordance with the "Principles of Laboratory Animal Care" formulated by the National Society for Medical Research and the "Guide for the Care and Use of
Laboratory Animals" prepared by the Institute of Laboratory Animal Resources of the National Research Council, published by the National Academy Press, revised in 2010.

\section{Data Collection and Measurements}

The operative data collected were the success rates of identifying accurate pulmonary segments. The identification of pulmonary segments was considered successful if it allowed for a complete transection of the intersegmental plane without injuries to the adjacent segmental vessels and bronchi. Therefore, the number of unexpected vascular and bronchial injuries was recorded. The quantity of injected vitamin $\mathrm{B}_{2}$ solution in each procedure was measured. The duration and light intensity of florescence in the target segment were also measured to provide a quantitative measure of success. The light intensity of florescence was measured at 15 -minute intervals after injection for 1 hour by using image analysis software U11437 (Hamamatsu Photonics, Hamamatsu, Japan). The light intensity of the adjacent segments was also measured for the sake of comparison. In addition, the intersegmental plane was analyzed in pathologic specimens stained with hematoxylin-eosin and azan.

\section{Statistical Analysis}

All data are expressed as mean \pm standard deviations. Comparative analyses were performed by Mann-Whitney $U$ tests using StatView software (SAS Institute, Inc, Cary, NC).

\section{RESULTS}

Overall, 20 segmentectomies were performed, 10 of each kind. In all procedures, it was possible to identify the target segment by the yellow-green fluorescence, both on the lung surface and inside the lung parenchyma. The mean quantity of injected liquid was $47.3 \pm 4.6 \mathrm{~mL}$ in cranial segmentectomy and $77.9 \pm 10.3 \mathrm{~mL}$ in $\mathrm{L} 2$ segmentectomy. The fluorescence in the intersegmental planes was easily identified, not only in simple cranial segmentectomy, but also in complicated L2 segmentectomy. No occurrences of unexpected injuries to the segmental bronchi and vessels or of incorrect identification of target segments were observed. The success rate of identifying accurate pulmonary segments was $100 \%$ in both procedures (Table 1).

The operative findings of each procedure are shown in Figures 3 and 4 . The right cranial lobe consists of a cranial and caudal segment; however, the intersegmental line cannot be recognized in normal light imaging (Figure 3, A). Fluorescence imaging revealed the intersegmental line clearly in both the ventral (Figure $3, B$ ) and dorsal aspects (Figure 3, C). A section along the intersegmental line reveals that the fluorescent intersegmental plane was easily recognizable, even inside the lung parenchyma (Figure 3,D and $E$ ). Transection of the intersegmental plane was completed without injury to the major segmental bronchi or vessels (Figure 3,F). The right L2 segment is adjacent to 3 other segments: L3, V2, and D2. The L2 segment could not be recognized by fluorescence imaging before vitamin $\mathrm{B}_{2}$ injection (Figure $4, A$ ), whereas fluorescence imaging after vitamin $B_{2}$ injection revealed the L2 segment clearly (Figure 4, B). Fluorescence imaging could also reveal each intersegmental plane clearly inside the lung 


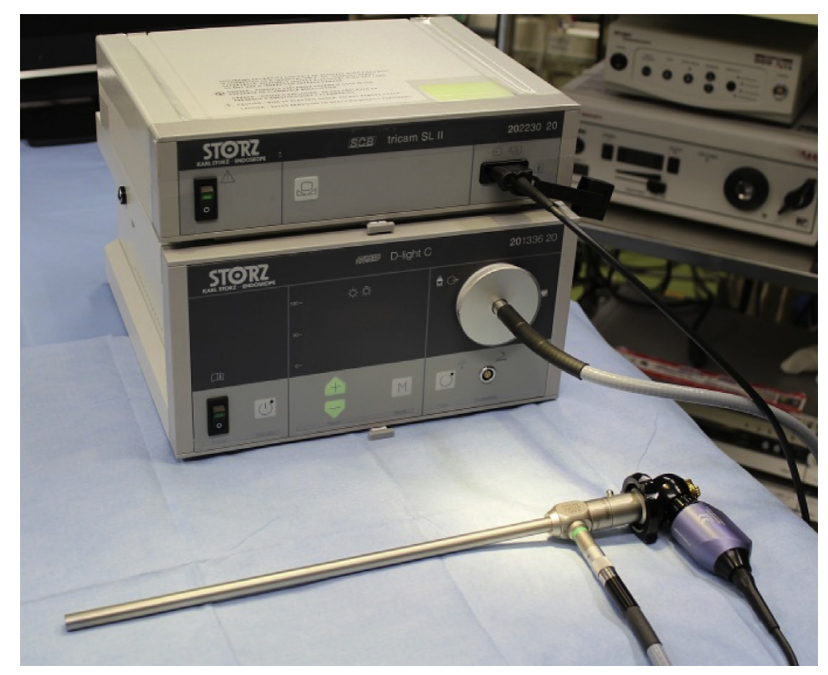

FIGURE 1. The PDD endoscope system.

parenchyma (Figure 4, $C$ to $E$ ). Cutting along the fluorescent plane allowed the segmental bronchus and the intersegmental pulmonary vein to be identified (Figure $4, D$ ). Even after transection of the lung parenchyma, fluorescence of the target segment continued without attenuation of light intensity (Figure $4, F$ ).

The light intensity of the target fluorescent segments was $148.1 \pm 11.9$ just after injection, $159.5 \pm 14.0$ after 15 minutes, $158.4 \pm 12.1$ after 30 minutes, and $158.1 \pm 13.5$ after 1 hour. For the adjacent nonfluorescent segments, the measured light intensity was $47.0 \pm 6.9$ just after injection, $50.8 \pm 5.8$ after 15 minutes, $54.0 \pm 5.5$ after 30 minutes, and $58.7 \pm 6.1$ after 1 hour. At every measurement from injection until 1 hour later, the light intensity of the target fluorescent segments was significantly stronger than that from the adjacent nonfluorescent segments (Figure 5).

Pathologic analysis revealed that the injected material remained only in the injected segment. It is noteworthy that the fibrous tissue of the intersegmental plane could be recognized in the azan-stained specimen (Figure 6, A and $B)$.

\section{DISCUSSION}

In this study, we developed and evaluated a novel fluorescence technique that involved transbronchial injection of vi$\operatorname{tamin} \mathrm{B}_{2}$ and the PDD endoscope system. This technique made it possible to identify the target pulmonary segment clearly and to perform accurate segmentectomies easily in ex vivo porcine lung.

In general, pulmonary segmentectomy is technically more complex than pulmonary lobectomy, requiring 3-dimensional knowledge of the relevant bronchovascular relationships and the possible anomalies of the vascular branches. Furthermore, identification of the intersegmental plane is essential to perform segmentectomy. It is usually difficult to identify the intersegmental plane without an imaging method. The most common method used is the air inflation-deflation technique. ${ }^{3,4}$ Although it is simple to identify the intersegmental plane on the lung surface using this technique, it may be difficult to identify the intersegmental plane inside the lung parenchyma while cutting the segment. Furthermore, during VATS or
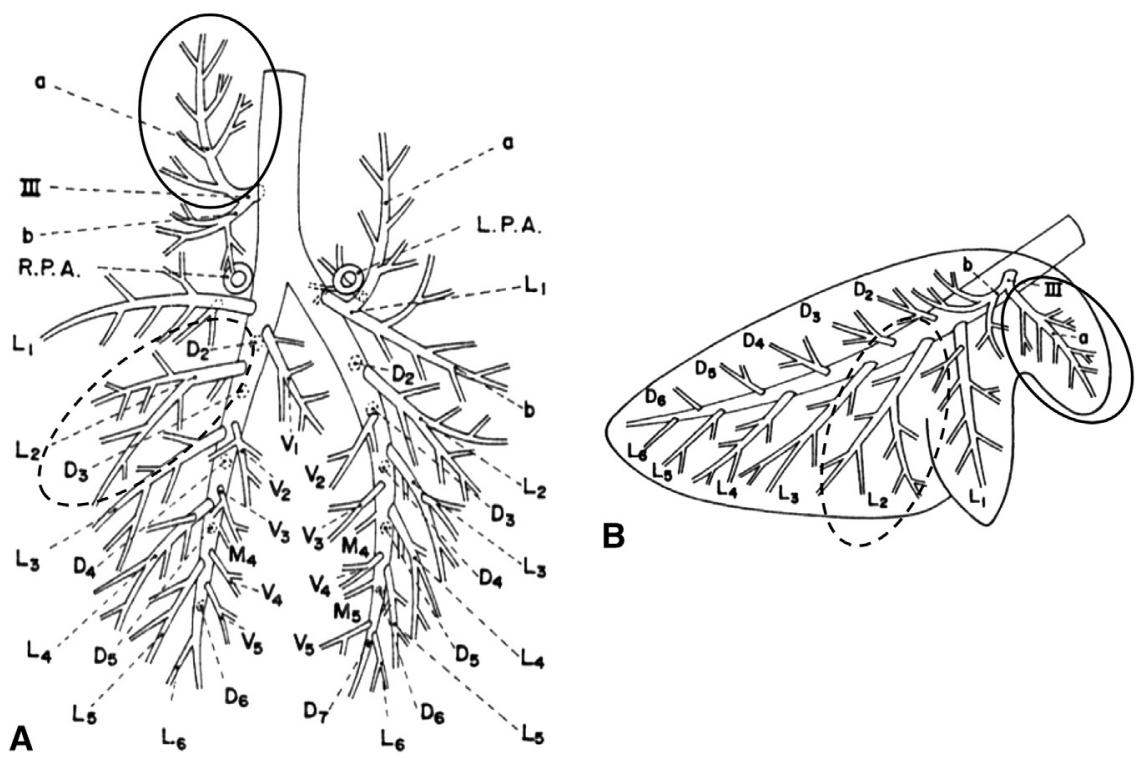

FIGURE 2. Schema of the porcine lung. A, Ventral aspect. B, Right lateral aspect. The right cranial lobe consists of only a cranial segment and a caudal segment. The right caudal lobe consists of lateral $(L)$ segments 2 to 6 , dorsal $(D)$ segments 2 to 6 , medial $(M)$ segment 4 , and ventral $(V)$ segments 2 to 5 . The right cranial segment is circled with a continuous line. The right L2 segment is circled with a dashed line. RPA, Right pulmonary artery; LPA, left pulmonary artery; $a$, cranial branch; $b$, caudal branch; III, cranial lobe bronchus. Reprinted with permission. ${ }^{10}$ 
TABLE 1. Segmentectomy

\begin{tabular}{lcc}
\hline & Cranial segmentectomy & L2 segmentectomy \\
\hline Procedures & 10 & 10 \\
Quantity of liquid $(\mathrm{mL})$ & $47.3 \pm 4.6$ & $77.9 \pm 10.3$ \\
Identification rate $(\%)$ & 100 & 100 \\
Vessel injury & 0 & 0 \\
Bronchial injury & 0 & 0 \\
\hline
\end{tabular}

L, Lateral.

minimal access open surgery, an inflated lung may obstruct the exposure and limit visualization of the target segment.

An alternative approach to segmental identification was suggested by Misaki and colleagues, ${ }^{5}$ who reported a novel method for determining adjacent lung segments by using infrared thoracoscopy. After ligation of the corresponding pulmonary artery of the target segment, indocyanine green (ICG) was administered intravenously during infrared thoracoscopy. This technique can identify the intersegmental line without lung inflation and, thus, the obstruction of surgical fields and the influence of emphysema may be minimized. However, the duration of intravascular ICG visualization is too short to perform segmentectomy. A large amount of ICG may be required in complicated segmentectomies. Furthermore, anatomic segmentectomy is ideally guided by the segmental bronchus, which is the most reliable landmark of a segment because of its rare anomaly. Another approach using ICG was suggested by Sekine and colleagues, ${ }^{6}$ who reported a novel method using transbronchial injection of ICG combined with infrared thoracoscopy. Although this technique may overcome some disadvantages of the previous method, a number of difficulties remain. The main risk of injecting ICG is allergy and tissue toxicity. ICG cannot be used for patients who have an iodine allergy, and undiluted or high concentrations of ICG can induce mucosal inflammation and epithelial injury. $^{11}$

An important feature of our technique is that it allows a very clear identification of the intersegmental plane owing to the strong fluorescent signal. This strong yellow-green fluorescence could reveal intersegmental planes, not only on the surface of the lung, but also inside the lung parenchyma. In addition, relatively little vitamin $\mathrm{B}_{2}$ solution was needed, thereby avoiding overexpansion of the target segment. Both these features make the approach potentially superior to the air inflation-deflation technique. Moreover, the yellow-green fluorescence used in this study provides not only light intensity, but also color information. The fluorescence observed with transbronchial injection of ICG using infrared thoracoscopy is similar but only provides information about light intensity. The duration of fluorescence using the method described in our study was more than 1 hour without any attenuation of light intensity,

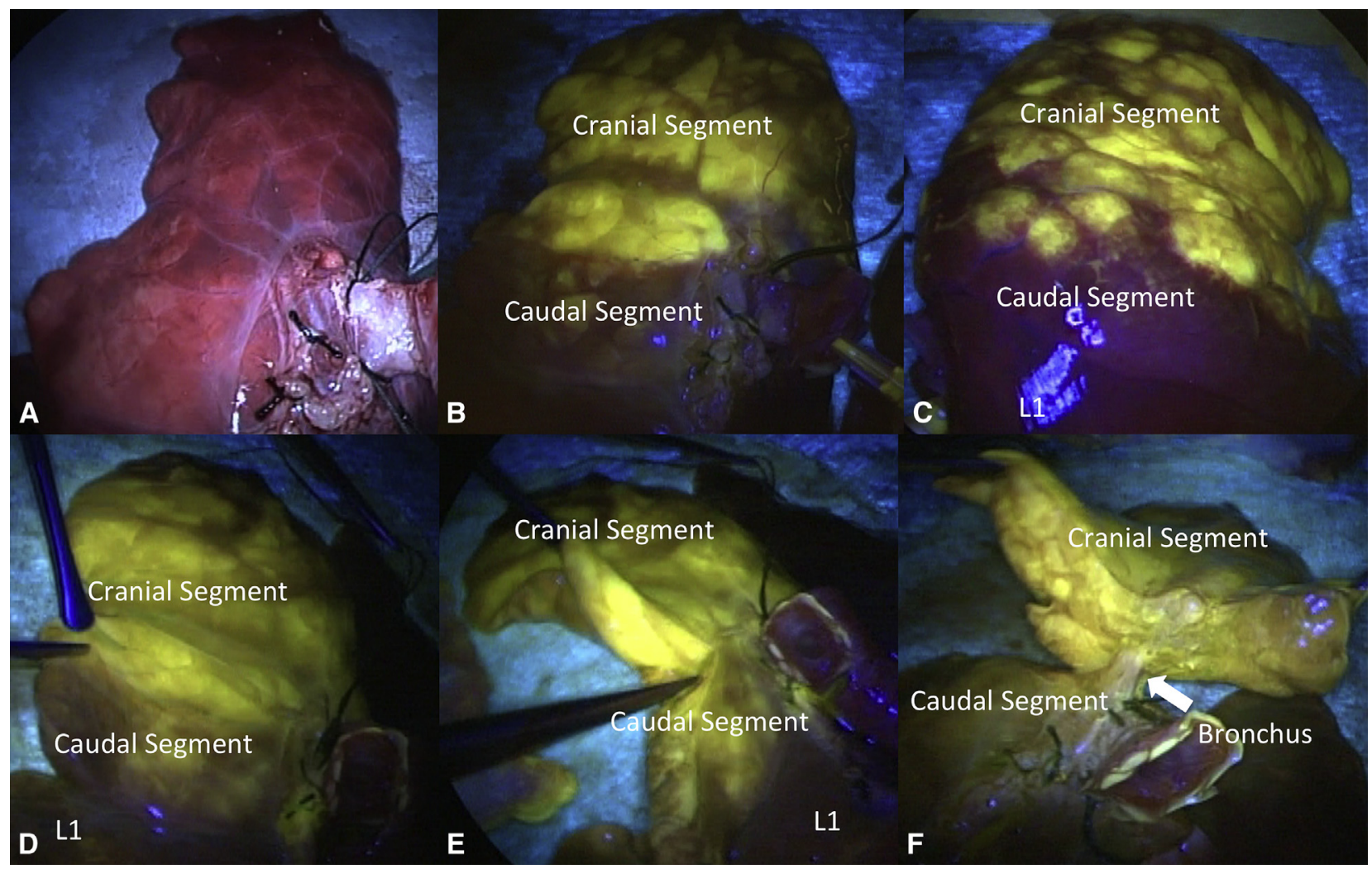

FIGURE 3. Segmentectomy of the right cranial lobe. A, Normal light imaging. B to F, Fluorescence imaging. L1, Lateral segment. 


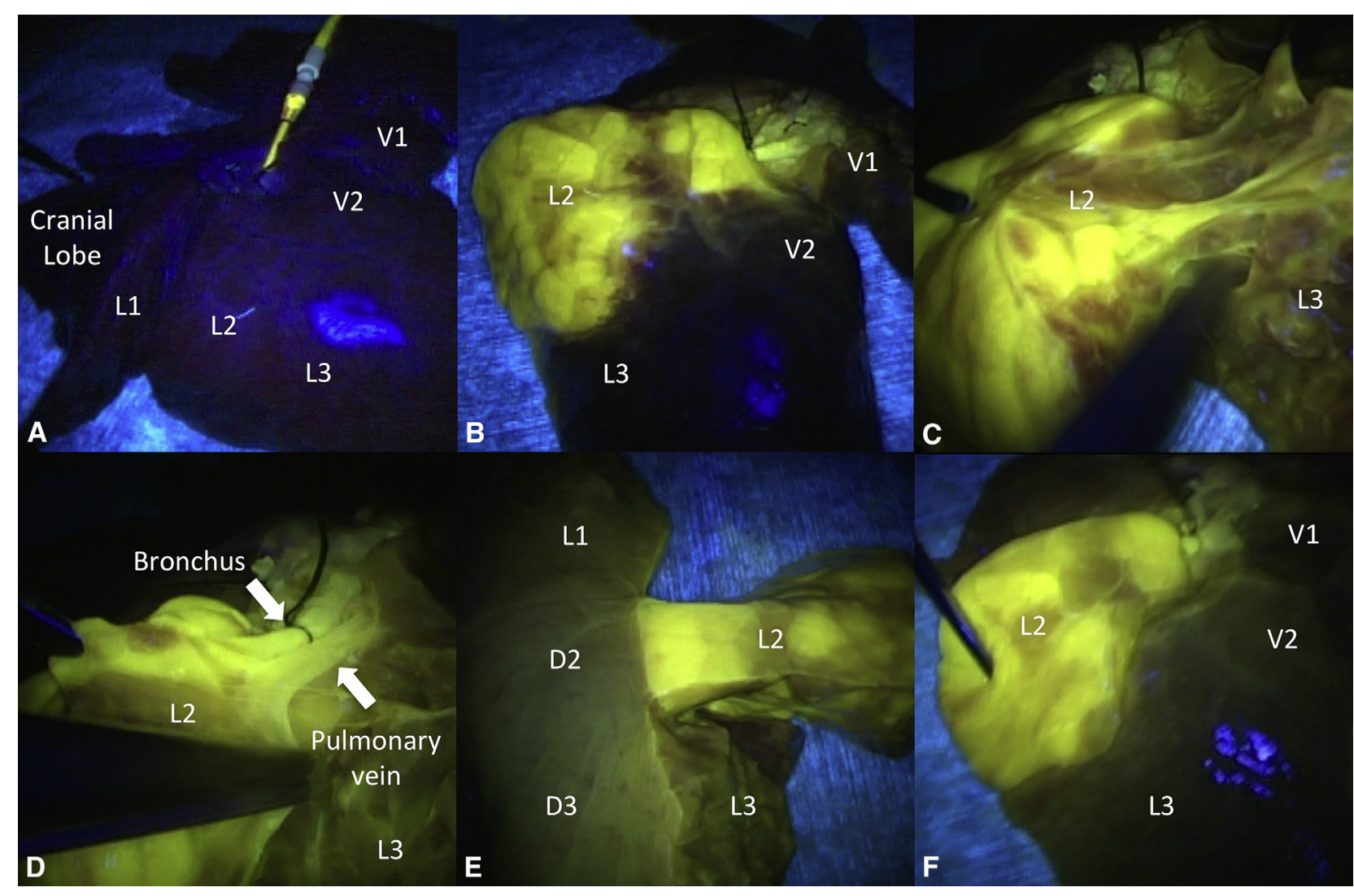

FIGURE 4. L2 segmentectomy in the right caudal lobe. A to F, Fluorescence imaging. $L$, Lateral segment; $V$, ventral segment; $D$, dorsal segment.

potentially providing enough time to transect the intersegmental plane.

Another advantage of our method is the safety of using vitamin $B_{2}$ as a fluorescence substance. Vitamin $B_{2}$ is a water-soluble vitamin that is necessary for the metabolism of fat, carbohydrate, and protein in the body; it is also required for cell respiration, formation of blood cells, production of antibodies, and normal growth. Vitamin $\mathrm{B}_{2}$ is also

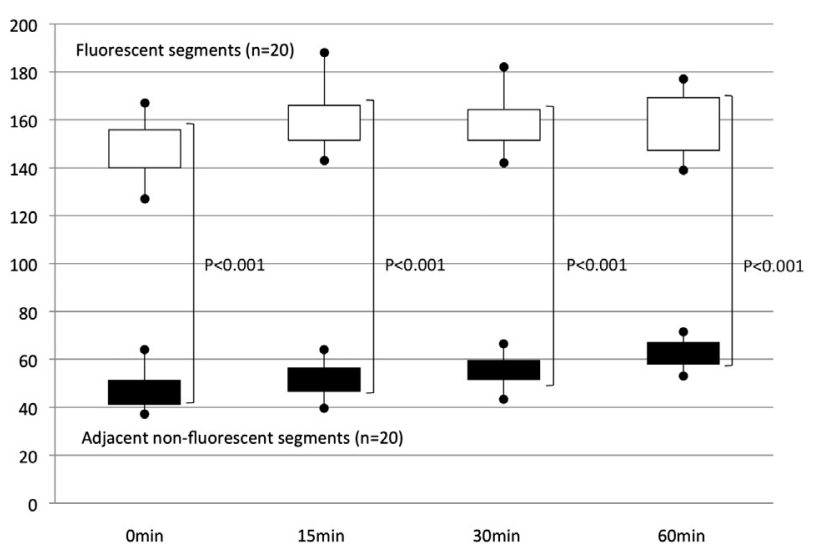

FIGURE 5. Comparison of light intensity between the fluorescent and adjacent nonfluorescent segments measured at 15 -minute intervals after injection until 1 hour later. maintained at a constant level in major organs, and any excess is eliminated in the urine. ${ }^{9}$ Furthermore, the diluted solution of FAD sodium is isotonic and neutral. These features greatly reduce the risk of allergic reaction, excessive damage, and tissue toxicity, a feature that represents a significant advantage over ICG. We have also confirmed the safety of injecting vitamin $B_{2}$ sodium into the lung in living animals. In 3 live pigs, $100-\mathrm{mL}$ vitamin $\mathrm{B}_{2}$ sodium injections into the lung were performed via bronchoscopy. After the pigs had been monitored for 2 weeks after injection, no adverse events were observed. An additional consideration is that vitamin $B_{2}$ is inexpensive; the cost of FAD sodium in each segmentectomy is less than $\$ 1.00$ in US currency. Further, the PDD endoscope system can perform not only fluorescence imaging, but also normal light imaging, and has almost the same specifications as the conventional thoracoscopic system. Therefore, the application of this technique to VATS should be straightforward.

The main limitation of the present study was that we used an ex vivo animal model and not human subjects; therefore, there is no assurance that the same results would be observed in human subjects. Of particular concern is the fact that the pathologic findings revealed fibrous tissue between the segments, which might be distinctive of porcine lungs. Therefore, the identification of the intersegmental plane in 


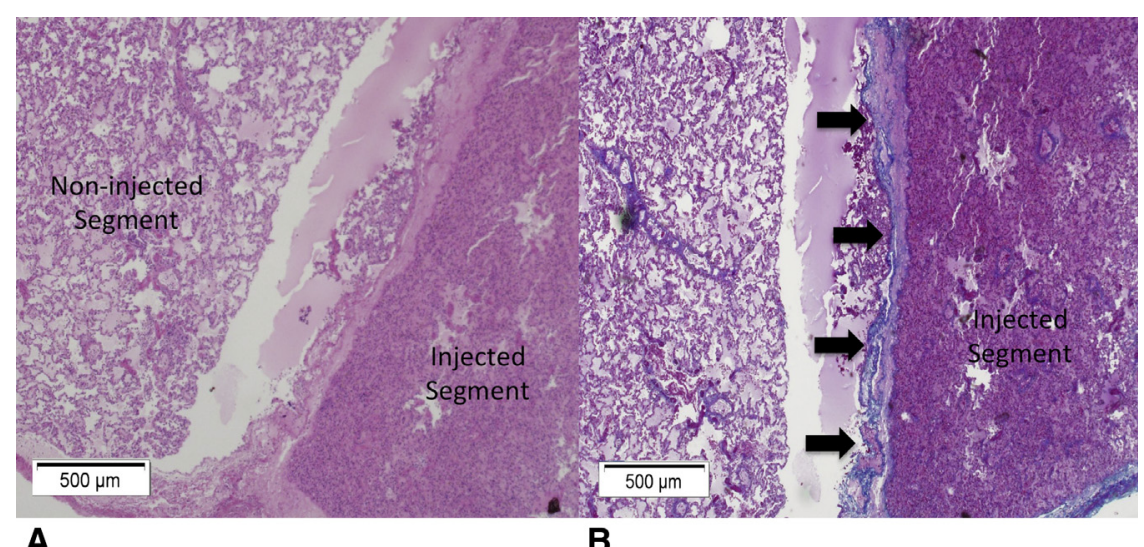

FIGURE 6. Pathologic findings of the intersegmental plane. A, Hematoxylin-eosin stain; B, Azan stain. Arrows point to the fibrous tissue in the intersegmental plane.

the porcine lung might be easier than that in humans. In this technique, transbronchial injection of vitamin $B_{2}$ was performed. We should consider the collateral, such as the pores of Kohn and Lambert tube, as well as the unfavorable effects of pulmonary emphysema in human clinical practice. These factors may impair the clear and precise identification of the intersegmental plane by fluorescence. However, these unfavorable effects may be minimal by using this technique compared with the air inflation-deflation technique because, as a liquid, vitamin $B_{2}$ is more difficult to spread than air. In emphysematous lungs, we prefer to cut the lung parenchyma with stapling devices to avoid postoperative air leak. Therefore, it is not necessary to be limited to making precise intersegmental planes in emphysema. From a technical aspect, slow and low-pressure injection of vita$\min B_{2}$ may minimize the effects. Furthermore, there is no circulation, ventilation, or metabolism in the ex vivo lung, and the effects of these in vivo would have to be considered. The duration of fluorescence may be shortened and the light intensity may be weakened in the in vivo lung. For the purpose of introducing this technique into human clinical practice, it will be necessary to clarify the potential problems in in vivo lungs to improve our technique. We have already begun to examine this technique in in vivo porcine lungs, and our preliminary results confirm that the duration and intensity of fluorescence was enough to perform pulmonary segmentectomy.

In conclusion, this fluorescence technique involving vi$\operatorname{tamin} \mathrm{B}_{2}$ and the PDD endoscope system allowed the identification of the pulmonary segment in ex vivo porcine lung. The advantages of this technique are clarity and safety, both of which are important in clinical practice. In the future, we will evaluate this technique in clinical trials.

We thank Professor A. Ooi for providing technical and educational support for lung pathology.

\section{References}

1. Okada M, Koike T, Higashiyama M, Yamato Y, Kodama K, Tsubota N. Radical sublobar resection for small-sized non-small cell lung cancer: a multicenter study. J Thorac Cardiovasc Surg. 2006;132:769-75.

2. Keenan RJ, Landreneau RJ, Maley RH Jr, Singh D, Macherey R, Bartley S, et al. Segmental resection spares pulmonary function in patients with stage I lung cancer. Ann Thorac Surg. 2004;78:228-33.

3. Tsubota N. An improved method for distinguishing the intersegmental plane of the lung. Surg Today. 2000;30:963-4.

4. Okada M, Mimura T, Ikegaki J, Katoh H, Itoh H, Tsubota N. A novel videoassisted anatomic segmentectomy technique: selective segmental inflation via bronchofiberoptic jet followed by cautery cutting. J Thorac Cardiovasc Surg. 2007; 133:753-8

5. Misaki N, Chang SS, Gotoh M, Yamamoto Y, Satoh K, Yokomise H. A novel method for determining adjacent lung segments with infrared thoracoscopy. J Thorac Cardiovasc Surg. 2009;138:613-8

6. Sekine Y, Ko E, Oishi H, Miwa M. A simple and effective technique for identification of intersegmental planes by infrared thoracoscopy after transbronchial injection of indocyanine green. J Thorac Cardiovasc Surg. 2012;143:1330-5.

7. Patel P, Bryan RT, Wallace MA. Emerging endoscopic and photodynamic techniques for bladder cancer detection and surveillance. Scientific World Journal. 2011;11:2550-8.

8. Matsumoto I, Ohta Y, Waseda R, Masaya T, Oda M, Watanabe G. Vitamin B $_{2}$ as a tracer for intraoperative pulmonary sentinel node navigation surgery. Anticancer Res. 2010;30:4109-14.

9. Konings EJ, Committee on Food Nutrition. Water-soluble vitamins. J AOAC Int. 2006;89:285-8.

10. Nakakuki S. Bronchial tree, lobular division and blood vessels of the pig lung. J Vet Med Sci. 1994;56:685-9.

11. Price N, Gottfried MR, Clary E, Lawson DC, Baillie J, Mergener K, et al. Safety and efficacy of India ink and indocyanine green as colonic tattooing agents. Gastrointest Endosc. 2000;51:438-42. 